Louisiana State University

LSU Digital Commons

Faculty Publications

Department of Physics \& Astronomy

$10-1-2016$

\title{
Efficacy of the SU(3) scheme for ab initio large-scale calculations beyond the lightest nuclei
}

\author{
T. Dytrych \\ Nuclear Physics Institute of the Academy of Sciences of the Czech Republic v. v. i. \\ P. Maris \\ lowa State University \\ K. D. Launey \\ Louisiana State University \\ J. P. Draayer \\ Louisiana State University \\ J. P. Vary \\ lowa State University
}

See next page for additional authors

Follow this and additional works at: https://digitalcommons.Isu.edu/physics_astronomy_pubs

\section{Recommended Citation}

Dytrych, T., Maris, P., Launey, K., Draayer, J., Vary, J., Langr, D., Saule, E., Caprio, M., Catalyurek, U., \& Sosonkina, M. (2016). Efficacy of the SU(3) scheme for ab initio large-scale calculations beyond the lightest nuclei. Computer Physics Communications, 207, 202-210. https://doi.org/10.1016/

j.cpc.2016.06.006

This Article is brought to you for free and open access by the Department of Physics \& Astronomy at LSU Digital Commons. It has been accepted for inclusion in Faculty Publications by an authorized administrator of LSU Digital Commons. For more information, please contact ir@lsu.edu. 


\section{Authors}

T. Dytrych, P. Maris, K. D. Launey, J. P. Draayer, J. P. Vary, D. Langr, E. Saule, M. A. Caprio, U. Catalyurek, and M. Sosonkina 


\title{
Efficacy of the SU(3) scheme for ab initio large-scale calculations beyond the lightest nuclei
}

\author{
T. Dytrych,${ }^{1,2, *}$ P. Maris,${ }^{3}$ K. D. Launey, ${ }^{2}$ J. P. Draayer,${ }^{2}$ J. P. Vary ${ }^{3}$ D. \\ Langr, ${ }^{4,5}$ E. Saule, ${ }^{6}$ M. A. Caprio, ${ }^{7}$ U. Catalyurek, ${ }^{8,9}$ and M. Sosonkina ${ }^{10}$ \\ ${ }^{1}$ Nuclear Physics Institute, Academy of Sciences of the Czech Republic, 25068 Rež, Czech Republic \\ ${ }^{2}$ Department of Physics and Astronomy, Louisiana State University, Baton Rouge, LA 70803, USA \\ ${ }^{3}$ Department of Physics and Astronomy, Iowa State University, Ames, IA 50011, USA \\ ${ }^{4}$ Faculty of Information Technology, Czech Technical University, Prague 16000, Czech Republic \\ ${ }^{5}$ Aerospace Research and Test Establishment, Prague 19905, Czech Republic \\ ${ }^{6}$ Department of Computer Science, University of North Carolina at Charlotte, NC 28223, USA \\ ${ }^{7}$ Department of Physics, University of Notre Dame, Notre Dame, IN 46556, USA \\ ${ }^{8}$ Department of Biomedical Informatics, The Ohio State University, Columbus, OH 43210, USA \\ ${ }^{9}$ Department of Electrical and Computer Engineering, \\ The Ohio State University, Columbus, OH 43210, USA \\ ${ }^{10}$ Department of Modeling, Simulation and Visualization Engineering, \\ Old Dominion University, Norfolk, VA 23529, USA
}

(Dated: June 11, 2016)

\begin{abstract}
We report on the computational characteristics of $a b$ initio nuclear structure calculations in a symmetry-adapted no-core shell model (SA-NCSM) framework. We examine the computational complexity of the current implementation of the SA-NCSM approach, dubbed LSU3shell, by analyzing $a b$ initio results for ${ }^{6} \mathrm{Li}$ and ${ }^{12} \mathrm{C}$ in large harmonic oscillator model spaces and $\mathrm{SU}(3)$-selected subspaces. We demonstrate LSU3shell's strong-scaling properties achieved with highly-parallel methods for computing the many-body matrix elements. Results compare favorably with complete model space calculations and significant memory savings are achieved in physically important applications. In particular, a well-chosen symmetry-adapted basis affords memory savings in calculations of states with a fixed total angular momentum in large model spaces while exactly preserving translational invariance.
\end{abstract}

PACS numbers: 21.60.Cs, 21.60.Fw, 21.60.De, 21.45.-v, 27.20.+n

\section{INTRODUCTION AND MOTIVATION}

In the last few years, ab initio approaches to nuclear structure and reactions have considerably advanced our understanding and capability of achieving first-principles descriptions of $p$-shell nuclei (see Refs. [1-9] and references therein). At the same time, fundamental approaches to the nucleon-nucleon $(N N)$ and three-nucleon $(N N N)$ interactions, such as meson-exchange theory and chiral effective field theory, have yielded major progress [10-15]. Successful realistic $N N$ interactions from inverse scattering have also emerged $[16,17]$. These new developments in microscopic nuclear theory combine to place serious demands on available computational resources for achieving converged properties of $p$-shell nuclei. This points to the need of further major advances in manybody methods to access a wider range of nuclei and experimental observables, while retaining the $a b$ initio predictive power.

These considerations motivate us to develop and investigate a novel model, the $a b$ initio symmetry-adapted no-core shell model (SA-NCSM) [18], which, by taking advantage of symmetries inherent to the nuclear dy-

*Corresponding author:dytrych@ujf.cas.cz namics [19-22], can provide access to heavier nuclei and larger model spaces essential to accommodate collective, deformed, and cluster substructures [22-32]. This is achieved by recognizing that the choice of a basis is crucial and that the SA-NCSM affords a solution that is a linear combination of a limited number of basis states of definite nuclear deformation. This yields memory savings in larger model spaces with fixed total angular momentum.

The concept underpinning the SA-NCSM has been demonstrated in our recent $a b$ initio studies of properties of ${ }^{6} \mathrm{Li},{ }^{6} \mathrm{He}$, and ${ }^{8} \mathrm{Be}[18,33]$. The potential gains from the SA-NCSM have also been demonstrated in our earlier study where we found that $a b$ initio wavefunctions of ${ }^{12} \mathrm{C}$ and ${ }^{16} \mathrm{O}$ calculated by the no-core shell model (NCSM) [4] project well onto a symmetry-adapted subspace that is only a tiny fraction of the corresponding complete model space [34-36]. While the SA-NCSM states can be obtained through a unitary transformation from the basis used in the NCSM, and hence span the entire space, the growth of the model space within the SA-NCSM framework can be managed, as shown here, by down-selecting to the physically relevant states as determined through symmetry considerations. This brings forward the major distinction as compared to the NCSM, namely, the selection of the SA-NCSM many-nucleon basis states retained in the calculation, which, in turn, allows the SA-NCSM to accommodate heavier nuclei and larger model spaces. 
Further advantages of the SA-NCSM include the capability of exactly removing the spurious center-of-mass motion within a selected subspace (thereby retaining this important factorization feature of the NCSM), as well as the use of efficient group-theoretical methods and coupling rules to build the many-nucleon basis states and interaction matrix elements.

In the present work, we study the efficacy of the SANCSM basis by comparing the complexity of calculations for SA-NCSM and NCSM, and we investigate the significance of selected SA-NCSM spaces against the corresponding NCSM (complete-space SA-NCSM) results. To achieve this, we evaluate properties of ${ }^{12} \mathrm{C}$ with both methods for comparable cutoffs. We adopt the JISP16 $N N$ interaction $[16,17]$ without renormalization and we neglect $N N N$ interactions. For both many-body methods, all $A$ nucleons in the nucleus are treated on the same footing. Observables, that can be experimentally measured, are obtained from $A$-nucleon wavefunctions resulting from Hamiltonian diagonalization in the chosen many-body model space. In particular, we consider the binding energies of the ground state $(g s)$ and eight lowlying excited states of positive parity in ${ }^{12} \mathrm{C}$. We also compare the $g s$ point-particle matter root-mean-square (rms) radius as well as the mass quadrupole moment of the first excited $2^{+}$and $4^{+}$states and the $B(E 2)$ electric quadrupole transition strength from the $2_{1}^{+}$to the ground state, together with the $B\left(M 1 ; 1_{1}^{+} \rightarrow 0_{\mathrm{gs}}^{+}\right)$transition strength and the $1_{1}^{+}$magnetic dipole moment.

It is worth noting that there are additional efforts aimed at accelerating the convergence of $a b$ initio nocore many-body methods using basis function techniques. The "Importance-Truncated" no-core shell model (ITNCSM) [8] attempts to sample the many-body configurations above a given cutoff using perturbative estimates of their contributions to the energy of low-lying states. The ab initio no-core Monte Carlo Shell Model (NC-MCSM) [37-39] aims to sample model spaces generated from an angular momentum and parity projected Hartree-Fock basis. Alternatively, the no-core full configuration approach (NCFC) represents an extrapolation to the infinite matrix limit of a sequence of calculations in finite model spaces and provides important observables, as some calculated quantities monotonically approach the exact result with increasing model spaces [40-43]. In this connection, there has been substantial recent progress in developing improved extrapolation techniques with quantitative uncertainty estimates [44-48].

Our goal is, first, to study the accuracy of SA-NCSM model space selections as compared to the completespace results, as well as the dependence of the results on the model space parameters for ${ }^{12} \mathrm{C}$ (Sec. III), and, then, to investigate the efficacy of the SA-NCSM by comparing the complexity of NCSM and SA-NCSM calculations (Sec. IV). This, in turn, will reveal the significance, in terms of potential memory savings for fixed- $J$ bases, of reducing the complete model space by searching for and retaining the physically relevant basis states important for the low-energy properties of nuclei with a realistic $N N$ interaction.

\section{QUANTUM MANY-BODY METHODS ADOPTED}

For a general problem, both NCSM and SA-NCSM adopt the intrinsic non-relativistic nuclear plus Coulomb interaction Hamiltonian defined as follows:

$$
H=T_{\text {rel }}+V_{N N}+V_{N N N}+\ldots+V_{\text {Coulomb }},
$$

where the $V_{N N}$ nucleon-nucleon and $V_{N N N} 3$-nucleon interactions are included along with the Coulomb interaction between the protons. The Hamiltonian may include additional terms such as multi-nucleon interactions among more than three nucleons simultaneously and higher-order electromagnetic interactions such as magnetic dipole-dipole terms.

In this study, the JISP16 NN interaction is adopted. It produces a high-quality description of the $N N$ scattering data and the deuteron $[16,17]$ as well as a good description of a range of properties of light nuclei [40]. We include the Coulomb interaction but we neglect higherorder electromagnetic interactions as well as $N N N$ interactions and beyond. We treat neutron and proton orbitals independently so total isospin is not conserved.

For each method, we retain many-nucleon basis states of a fixed parity, consistent with the Pauli principle, and limited by a many-body basis cutoff $N_{\max }$. The $N_{\max }$ cutoff is defined as the maximum number of harmonic oscillator (HO) quanta allowed in a many-nucleon basis state above the minimum for a given nucleus. We seek to obtain the lowest few eigenvalues and eigenfunctions of the Hamiltonian (1). The resulting calculated $g s$ energy is a rigorous upper bound on the exact result for the full (infinite) basis and monotonically approaches the exact result as the $N_{\max }$ increases. This upper bound character applies to the lowest calculated state of each total angular momentum and parity. We defer extrapolations to exact infinite matrix results to other efforts such as Refs. [4045].

The NCSM calculations may be performed in an $M$ scheme basis where the many-nucleon basis states are constructed with a good total magnetic projection $M$ that is the same for all basis states ( $M=0$ here). The eigensolutions have good total angular momentum up to numerical errors and this serves as a cross-check on the precision of the calculations. Note that we employ a Lanczos scheme that selectively converges the low-lying solutions, regardless of their total angular momenta, in the same $M$-scheme calculation, thus facilitating the identification of the ground-state spin as well as calculations of electromagnetic transition rates. For the NCSM $M$-scheme calculations reported here, we employ the code "Many-Fermion Dynamics - nuclear" or "MFDn" [49] which has been revised and optimized for leadershipclass parallel computers [50-53]. 
The NCSM calculations may also be performed in a $J$-scheme basis where the many-nucleon basis states are constructed with a good total angular momentum $J$ and total magnetic projection $M$ that is the same for all basis states [54]. This approach again uses the Lanczos procedure. Several runs are then needed to map out all the states in the low-lying spectrum. As the SA-NCSM utilizes basis states with a good $J$ quantum number, we use the $J$-scheme NCSM approach for the primary reference case in order to compare computational complexity. It is useful to note, for example, that one may need a fixed$J$ scheme to isolate members of excited rotational bands that reside in a dense spectrum since the Lanczos procedure in the $M$ scheme is less efficient for these states.

For the SA-NCSM calculations, we work in a manynucleon basis labeled by the SU(3) quantum numbers $(\lambda \mu)$ of the Elliott model $[19,20]$, orbital momentum $L$, proton, neutron, and total intrinsic spins $\left(S_{p}, S_{n}\right.$, and $\left.S\right)$, as well as total angular momentum $J$ and its projection $M$ (similarly to NCSM, $M=0$ here). We employ a new code, "LSU3shell" [55], implemented using C++ and Fortran 77 programming languages and parallelized with a hybrid MPI/OpenMP approach, that runs efficiently on parallel computers, as shown in this paper.

\section{A. Model Space Parameters: $N_{\max }$ and $\hbar \Omega$}

All solutions have a dependence on the cutoff $N_{\max }$ and on the HO energy $\hbar \Omega$. The degree to which we obtain results independent of the $N_{\max }$ and of $\hbar \Omega$ is a measure of the convergence of the results since fully converged results are independent of both basis parameters.

For both methods, we employ the many-body $N_{\max }$ truncation where we enumerate all many-body states, with the selected symmetries, possessing total HO excitation quanta less than or equal to $N_{\max }$. Specifically, each single-particle state in a many-nucleon basis state contributes $2 n+l$ to the total HO quanta ( $n$ is the radial quantum number and $l$ is the orbital angular momentum quantum number) for that basis state. Then, the minimum sum for a given nucleus (of the lowest allowed configuration) is subtracted to give the total $\mathrm{HO}$ excitation quanta. The smallest model space for each nucleus is then $N_{\max }=0$ and increases in units of 2 for the states of the same parity. Odd values of $N_{\max }$ cover the states with opposite parity. With this scheme, basis states where one nucleon carries all the $N_{\max }$ quanta are included, in which cases one nucleon occupies the highest HO shell.

In both NCSM and SA-NCSM, the $N_{\max }$ cutoff in the $\mathrm{HO}$ basis is valuable for preserving Galilean invariance. That is, with the $N_{\max }$ cutoff, we guarantee that all solutions factorize into a product of intrinsic and centerof-mass motion components. With a Lagrange multiplier term acting on the center-of-mass [4, 56-58], we remove states of center-of-mass excitation from low-lying spectrum. All observables may then be evaluated free of spu- rious center-of-mass motion contributions.

\section{B. Ab initio SA-NCSM}

The SA-NCSM framework allows one to down-select from all possible configurations to a subset that tracks with an inherent preference of a system towards low-spin and high-deformation dominance - and symplectic multiples thereof in high- $N_{\max }$ spaces [18] - as revealed to be important in realistic NCSM wavefunctions [34-36].

The many-nucleon basis states of the SA-NCSM are decomposed into spatial and intrinsic spin parts, where the spatial part is further classified according to the $\mathrm{SU}(3) \supset \mathrm{SO}(3)$ group chain. The significance of the $\mathrm{SU}(3)$ group for a microscopic description of the nuclear collective dynamics can be seen from the fact that it is the symmetry group of the successful Elliott model [19, 20], and a subgroup of the physically relevant $\operatorname{Sp}(3, \mathbb{R})$ symplectic model [21, 22], which provides a comprehensive theoretical foundation for understanding the dominant symmetries of nuclear collective motion. The SA-NCSM basis states are labeled schematically as

$$
\left|\vec{\gamma} ; N(\lambda \mu) \kappa L ;\left(S_{p} S_{n}\right) S ; J M\right\rangle
$$

where $S_{p}, S_{n}$, and $S$ denote proton, neutron, and total intrinsic spins, respectively, $N$ is the total number of $\mathrm{HO}$ excitation quanta, and $(\lambda \mu)$ represent a set of quantum numbers that labels an SU(3) irreducible representation, or "irrep" 1 The label $\kappa$ distinguishes multiple occurrences of the same orbital momentum $L$ in the parent irrep $(\lambda \mu)$. The $L$ is coupled with $S$ to the total angular momentum $J$ and its projection $M$. The symbol $\vec{\gamma}$ schematically denotes the additional quantum numbers needed to specify a distribution of nucleons over the major HO shells and their single-shell and inter-shell quantum numbers. Specifically, in each major HO shell $\eta$ with degeneracy $\Omega_{\eta}$, protons (or neutrons) are arranged into antisymmetric $\mathrm{U}\left(\Omega_{\eta}\right) \times \mathrm{SU}(2)_{S_{\eta}}$ irreps [62], with $\mathrm{U}\left(\Omega_{\eta}\right)$ further reduced with respect to $\mathrm{SU}(3)$, providing the single-shell labels $\left[f_{1}, \ldots, f_{\Omega_{\eta}}\right] \alpha_{\eta}\left(\lambda_{\eta} \mu_{\eta}\right) S_{\eta}$. Note that a spatial symmetry associated with a Young tableau $\left[f_{1}, \ldots, f_{\Omega_{\eta}}\right]$ is uniquely determined by the imposed antisymmetrization and the associated intrinsic spin $S_{\eta}[62]$. A multiplicity index $\alpha_{\eta}$ is required to distinguish multiple occurrences of $\mathrm{SU}(3)$ irrep $\left(\lambda_{\eta} \mu_{\eta}\right)$ in a given $\mathrm{U}\left(\Omega_{\eta}\right)$ irrep. Coupling of these single-shell configurations further yield inter-shell $\mathrm{SU}(3) \times \mathrm{SU}(2)_{S}$ quantum numbers for protons and for neutrons; the proton and neutron configurations are finally coupled to good $(\lambda \mu) \kappa L S ; J M$. All of these

1 The SU(3) irrep labels $(\lambda \mu)$ bring forward important information about nuclear shapes and deformation, according to an established mapping [59-61]. For example, $(00),(\lambda 0)$ and $(0 \mu)$ describe spherical, prolate and oblate deformation, respectively. 


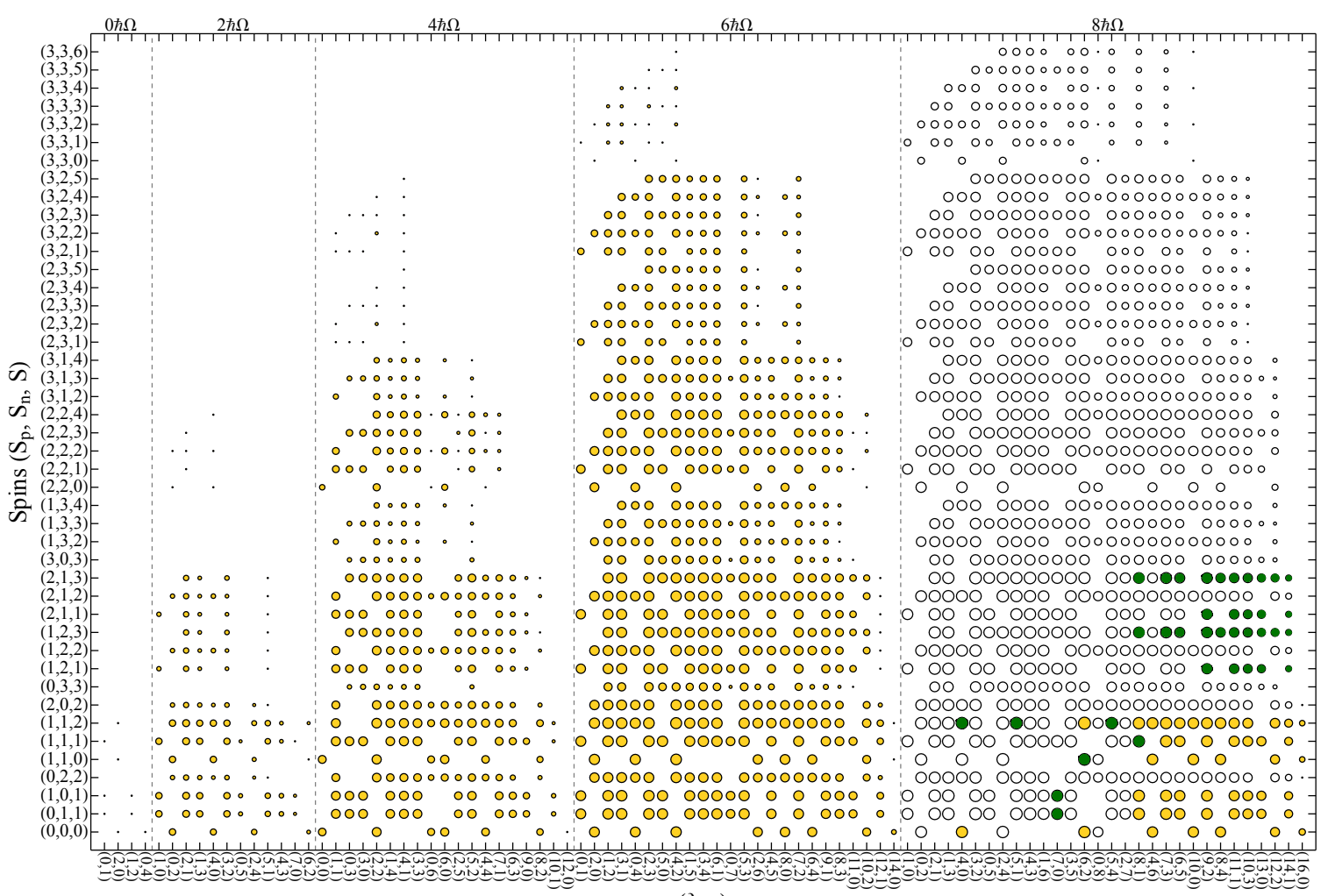

$(\lambda \mu)$

FIG. 1: Complete $J=0$ model space for ${ }^{12} \mathrm{C}$ and $N_{\max }=8$ given in terms of proton $\left(S_{p}\right)$, neutron $\left(S_{n}\right)$ and total $(S)$ spin values (vertical axis) and deformation $(\lambda \mu)$ (horizontal axis). Each circle represents basis states carrying the same $\left(S_{p}, S_{n}, S\right)$ and $(\lambda \mu)$ quantum numbers within a $\hbar \Omega$-subspace, with the radius being proportional to $\log _{10}$ of the number of such states. Filled light yellow circles indicate the $\langle 6\rangle$ 8-A restricted model space employed by the SA-NCSM for calculations of $0^{+}$states in ${ }^{12} \mathrm{C}$, while all filled circles indicate the $\langle 6\rangle 8-\mathrm{B}$ restricted model space. Similar selection rules are applied to higher- $J$ states.

labels uniquely determine the SA-NCSM basis states (2).

To accommodate highly-deformed configurations with high-energy HO excitations together with essential mixing of low-energy excitations, typical SA-NCSM calculations span the entire (complete) space up to a given $N_{\max }^{\perp}$, while beyond this, calculations include only selected many-nucleon basis states limited by the $N_{\max }$ cutoff $[18,33,63]$. We adopt a notation where an SANCSM model space of " $\left\langle N_{\max }^{\perp}\right\rangle N_{\max }$ " includes all the configurations up through $N_{\max }^{\perp}$ and a restricted subspace beyond $N_{\max }^{\perp}$ up through $N_{\max }$. For example, the selections of basis states employed in this paper for a $\langle 6\rangle 8$ model space, " $\langle 6\rangle 8-\mathrm{A}$ " and " $\langle 6\rangle 8$-B", are illustrated for ${ }^{12} \mathrm{C}$ and $J=0$ states $^{2}$ in Fig. 1. The $\langle 6\rangle 8$ space includes all configurations up through $6 \hbar \Omega$ with the additional restriction of the $8 \hbar \Omega$ subspace to only selected deformations as well as selected proton spin, neutron spin, and total spin values as shown in Fig. 1. Namely,

${ }^{2}$ Lists of the resulting configurations for other $J$-values are available upon request.
$\left(S_{p}, S_{n}, S\right)=(0,0,0),(0,1,1),(1,0,1),(1,1,0),(1,1,1)$ and $(1,1,2)$. A similar selection pattern is employed for $J=1,2$ and $J=4$. The selection is based on highdeformation and low-spin dominance, along with symplectic $\operatorname{Sp}(3, \mathbb{R})$ excitations thereof. Hence, configurations of largest deformation (typically, large $\lambda$ and $\mu$ ) and lowest spin values are included first, that is, the lower right corner of the $8 \hbar \Omega$ subspace in Fig. 1. The mathematical prescription for retaining states in these model spaces, together with the associated quantitative cutoff, is based on equations and conditions expressed within the $\mathrm{SU}(3)$ and $\operatorname{Sp}(3, \mathbb{R})$ groups [63] that are beyond the scope of this presentation.

A very important feature of the SA-NCSM is that any SA-NCSM selected model space of $\mathrm{SU}(3) \times \mathrm{SU}(2)_{S}$ irreps, that is, a space spanned by all configurations carrying a fixed set of $S_{p} S_{n} S$ and $N(\lambda \mu)$ quantum numbers, permits exact factorization of the center-of-mass motion [57]. As a result, a reduced model space $\left\langle N_{\max }^{\perp}\right\rangle N_{\max }$, defined by a set of physically relevant $(\lambda \mu)$ and important intrinsic spins, yields eigenfunctions that exactly factorize into a product of intrinsic and center-of-mass components. With the help of a Lagrange multiplier term, the wavefunctions, and associated observables, for states 
of interest (those free of center-of-mass excitation) are generated efficiently.

\section{III. ${ }^{12} \mathrm{C}$ PROPERTIES AND MODEL-SPACE SELECTION CONSIDERATIONS}

To examine the applicability of the SA-NCSM, we select the ground state and eight low-lying positive-parity excited states of ${ }^{12} \mathrm{C}$. Namely, three $0^{+}$states, three excited $2^{+}$states, an excited $4^{+}$state, as well as two excited $1^{+}$states. We evaluate and compare results for the binding energy, excitation energies, the ground-state pointparticle matter rms radius, the electric quadrupole moment of the $2_{1}^{+}$and $4_{1}^{+}$states as well as the $B\left(E 2 ; 2_{1}^{+} \rightarrow\right.$ $\left.0_{\mathrm{gs}}^{+}\right)$, the $B\left(M 1 ; 1_{1}^{+} \rightarrow 0_{\mathrm{gs}}^{+}\right)$transition strengths to the ground state and the magnetic dipole moment of the first excited $J=1, T=1$ state.

TABLE I: ${ }^{12} \mathrm{C}$ observables for selected $N_{\max }$ values, namely, binding energy BE $(\mathrm{MeV})$, excitation energies $E(\mathrm{MeV})$, electric quadrupole moments $Q\left(\mathrm{e} \mathrm{fm}^{2}\right)$ and magnetic dipole moment $\mu\left(\mu_{N}\right)$, as well as $B(E 2)\left(e^{2} \mathrm{fm}^{4}\right)$ and $B(M 1)\left(\mu_{N}^{2}\right)$ reduced transition strengths, together with point-particle matter rms radius $r_{m}(\mathrm{fm})$. The observables are calculated for $\hbar \Omega=20 \mathrm{MeV}$ using the bare JISP16 interaction and compared to the experiment ("Expt.") [64]. The SA-NCSM results are obtained in a reduced $\langle 6\rangle 8$ model space with a complete space up to $6 \hbar \Omega$ ("B" selection). Comparing MFDn and LSU3shell results up through $N_{\max }=6$ shows they are the same, to within the quoted precision.

\begin{tabular}{l|lllll}
\hline \hline & \multicolumn{5}{|c}{$N_{\max }$} \\
& Expt. & 4 & 6 & 8 & $\langle 6\rangle 8-\mathrm{B}$ \\
\hline $\mathrm{BE}$ & 92.162 & 72.654 & 82.192 & 87.902 & 85.951 \\
$E_{2_{1}^{+}}$ & 4.439 & 6.415 & 5.356 & 4.685 & 4.644 \\
$E_{1_{1}^{+}}$ & 12.71 & 17.024 & 15.307 & 14.161 & 14.199 \\
$E_{4_{1}^{+}}$ & 14.083 & 20.071 & 17.854 & 16.255 & 16.324 \\
$Q_{2_{1}^{+}}$ & $+6(3)$ & 3.316 & 3.546 & 3.741 & 3.735 \\
$Q_{4_{1}^{+}}$ & $\mathrm{N} / \mathrm{A}$ & 4.285 & 4.597 & 4.864 & 4.845 \\
$\mu_{1}^{+}$ & $\mathrm{N} / \mathrm{A}$ & 0.948 & 0.876 & 0.848 & 0.839 \\
$B\left(E 2 ; 2_{1}^{+} \rightarrow 0_{\mathrm{gs}}^{+}\right)$ & $7.59(42)$ & 2.723 & 3.051 & 3.342 & 3.301 \\
$B\left(M 1 ; 1_{1}^{+} \rightarrow 0_{\mathrm{gs}}^{+}\right)$ & $0.0145(21)$ & 0.028 & 0.018 & 0.013 & 0.012 \\
$r_{m}\left(0_{\mathrm{gs}}^{+}\right)$ & $2.43(2)^{a}$ & 1.996 & 1.995 & 2.003 & 2.005 \\
\hline \hline
\end{tabular}

${ }^{a}$ Ref. [65]

We aim to study here model-space selection considerations in the SA-NCSM by comparing results to the NCSM calculations for the same $N_{\max }$ cutoff. We consider two SU(3)-based selection schemes, determined by symmetry considerations, namely, " $\langle 6\rangle 8-\mathrm{A}$ " (a smaller set of basis states, Fig. 1, filled circles, light yellow colors) and " $\langle 6\rangle 8$-B" (a larger set of basis states, Fig. 1, filled circles), with dimensions listed in Table II and discussed in Sec. IV.

We present the $g s$ energy of ${ }^{12} \mathrm{C}$ obtained with MFDn in the $M$-scheme basis using the bare JISP16 interaction in Fig. 2 through a sequence of $N_{\max }$ truncations and as a function of $\hbar \Omega$. The energy converges uniformly from above as expected with increasing $N_{\max }$ and the curves become increasingly independent of $\hbar \Omega$ (i.e. flatter) with increasing $N_{\max }$. Both of these features are signals of $g s$ energy convergence. We obtain significant increases in binding with each increment in $N_{\max }$. The experimental result is indicated as a horizontal line. For completeness, we also show the NCFC extrapolated result (extrapolation method "A" of Ref. [40]) based on the results through $N_{\max }=10[42,43]$.

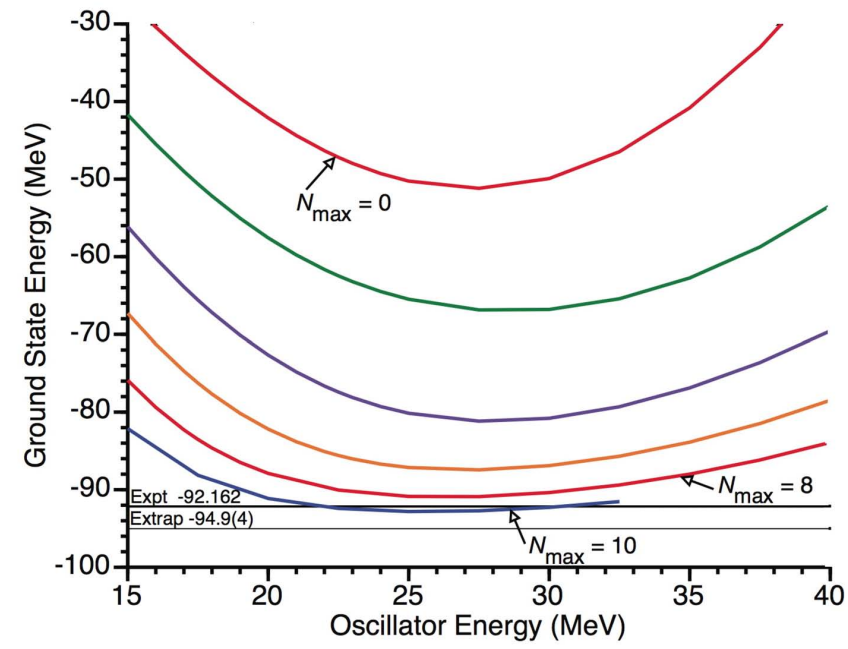

FIG. 2: Ground state energy of ${ }^{12} \mathrm{C}$ as a function of $\hbar \Omega$ for a sequence of $N_{\max }$ cutoffs with the JISP16 $N N$ interaction. The quoted NCFC extrapolated energy is obtained using extrapolation method "A" of reference [40].

In Table I, we compare the results for ${ }^{12} \mathrm{C}$ observables at $\hbar \Omega=20 \mathrm{MeV}$ obtained with MFDn (see also [66]) and LSU3shell at the $\mathrm{SU}(3)$ selection scheme " $\langle 6\rangle$ 8-B" mentioned above and discussed in Sec. IV below. Table I reveals the remarkable result that only $14 \%$ of the $J=0$ $8 \hbar \Omega$ subspace included in $\langle 6\rangle 8$-B $(2,716,280 / 18,741,257$ $=0.14$, with $18,741,257$ the dimension of the $J=08 \hbar \Omega$ subspace) provide $66 \%$ of the complete $N_{\max }=8$ modelspace increase in binding energy with respect to the $N_{\max }=6$ outcome. Overall the binding energy in $\langle 6\rangle$ 8-B reproduces $98 \%$ of the $N_{\max }=8$ NCSM binding energy. In fact, the $\langle 4\rangle 8$-B model space already reproduces $96 \%$ of this observable. This points to the fact that complete $0 \hbar \Omega, 2 \hbar \Omega$, and $4 \hbar \Omega$ model spaces $\left(N_{\max }^{\perp}=4\right)$ and only selected physically relevant $6 \hbar \Omega$ and $8 \hbar \Omega$ basis states suffice to capture most of the physics that governs the ${ }^{12} \mathrm{C}$ ground state. Further improvements are observed in the case of $\langle 6\rangle$ 8-B, but these improvements are less significant for various observables. In Table I, we also provide the experimental counterparts and note that, in general, meaningful comparisons between computations and experiment require both convergence with $N_{\max }$ and $N_{\max }^{\perp}$, along with quantification of numerical uncertainties due 
to basis truncations.

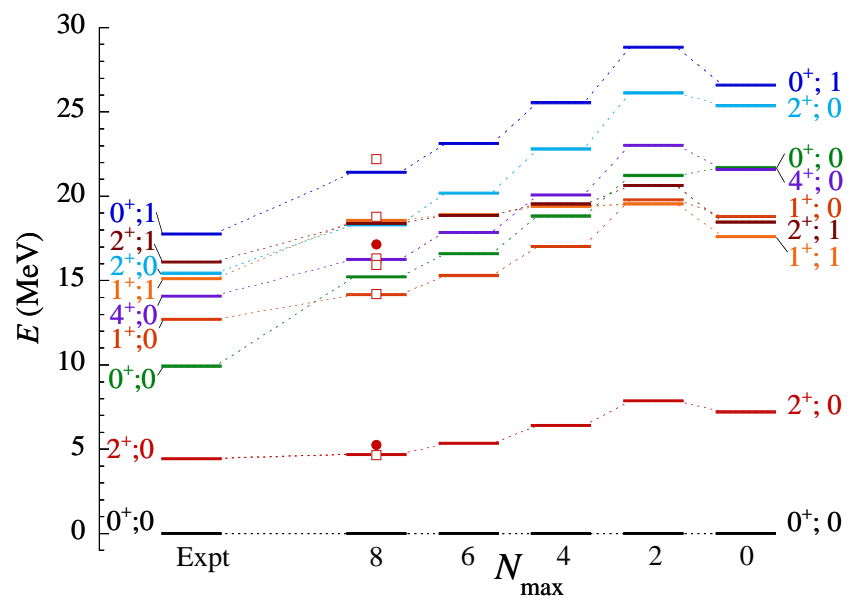

FIG. $3:{ }^{12} \mathrm{C}$ eigenenergies obtained with the MFDn and the bare JISP16 interaction for $\hbar \Omega=20 \mathrm{MeV}$ as a function of $N_{\max }$. The corresponding SA-NCSM results for $\langle 6\rangle 8$ are shown for the "A" (filled circles) and "B" (empty squares) selection schemes; the order of the empty squares is as follows, from bottom to top, $2_{1}^{+}\left(2^{+} ; 0\right), 1_{1}^{+}\left(1^{+} ; 0\right), 0_{3}^{+}\left(0^{+} ; 0\right), 4_{1}^{+}\left(4^{+} ; 0\right)$, $2_{2}^{+}\left(2^{+} ; 0\right)$, and $0_{4}^{+}\left(0^{+} ; 1\right)$. Note that the $7.65-\mathrm{MeV} 0_{2}^{+}$state (the so-called Hoyle state) is not included in the plot.

To study convergence of excitation energies, we present the low-lying spectrum of ${ }^{12} \mathrm{C}$ at $\hbar \Omega=20 \mathrm{MeV}$ as a function of $N_{\max }$ (Fig. 3). The outcome reveals that with increasing $N_{\max }$, the difference between the theoretical and experimental excitation energies is seen to decrease. The $\langle 6\rangle$ 8-B SA-NCSM calculations for the isospin-zero $2_{1}^{+}, 1_{1}^{+}$, and $4_{1}^{+}$excited states (Table I and Fig. 3, empty squares) deviate from the corresponding $N_{\max }=8$ results only by $0.9 \%, 0.3 \%$, and $0.4 \%$, respectively. Higherlying states, such as $0_{3}^{+}, 2_{2}^{+}$, and $0_{4}^{+}$, exhibit a slightly larger deviation, with the largest difference observed for $0_{4}^{+}$, which is still only about $800 \mathrm{keV}$. These states are found to lie remarkably close to the complete-space counterparts even when the smaller $\langle 6\rangle$ 8-A SA-NCSM model space is utilized (see Fig. 3 for $2_{1}^{+}$and $4_{1}^{+}$, filled circles). In addition, we compare excitation energies as a function of $\hbar \Omega$ in the range of 15 to $25 \mathrm{MeV}$. In this region, we find that restricted SA-NCSM model spaces yield excitation energies that change only slightly with $\hbar \Omega$, e.g., for the $2_{1}^{+}$and $4_{1}^{+} T=0$ states, the energies change by less than $1 \mathrm{MeV}$ through a change of $10 \mathrm{MeV}$ in the $\hbar \Omega$ oscillator energy (Fig. 4).

Table I also presents restricted-space and completespace results for the electric quadrupole moment of the first excited $2^{+}$and $4^{+}$states of ${ }^{12} \mathrm{C}$, the reduced probability for the $E 2$ transition of $2_{1}^{+}$and $M 1$ transition of $1_{1}^{+}$to the ground state, as well as the point-nucleon matter rms radius of the ground state. These observables are remarkably well reproduced by the SA-NCSM calculations in the $\langle 6\rangle 8$-B space. Similar results are obtained already in the $\langle 4\rangle 8$-B model space. For example, $B\left(E 2 ; 2_{1}^{+} \rightarrow 0_{\mathrm{gs}}^{+}\right)=3.339 e^{2} \mathrm{fm}^{4}$, which represents $99.9 \%$

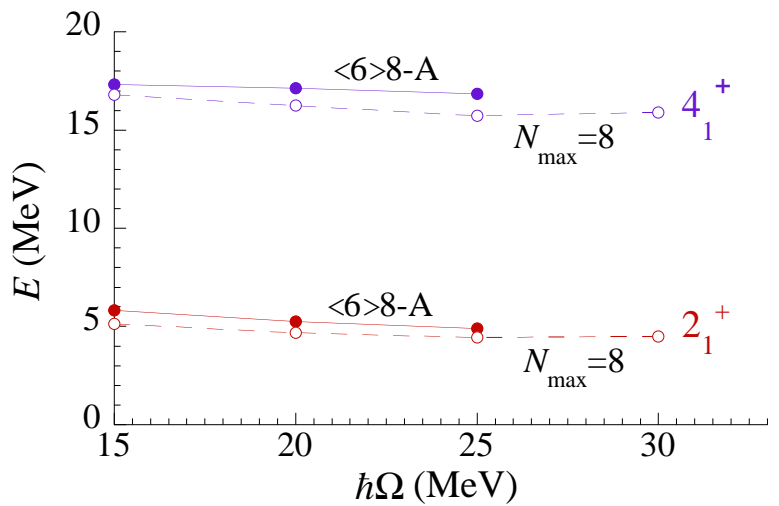

FIG. $4:{ }^{12} \mathrm{C}$ eigenenergies as a function of $\hbar \Omega$ obtained with the SA-NCSM and the bare JISP16 interaction for the $\langle 6\rangle 8$ A selection scheme (filled circles, solid lines), and compared to the complete $N_{\max }=8$ model space (open circles, dashed lines).

of the complete space result. The smaller $\langle 6\rangle$ 8-A model space yields, $Q_{2_{1}^{+}}=3.712 \mathrm{efm}^{2}, Q_{4_{1}^{+}}=4.826 \mathrm{efm}^{2}$, $B\left(E 2 ; 2_{1}^{+} \rightarrow 0_{\mathrm{gs}}^{+}\right)=3.289 e^{2} \mathrm{fm}^{4}$, and $r_{m}\left(0_{\mathrm{gs}}^{+}\right)=2.007 \mathrm{fm}$, which already reproduce more than $99 \%$ of the $N_{\max }=8$ NCSM results. This indicates that these observables are not sensitive to the fine-tuning of the selected space and only a few configurations of the $8 \hbar \Omega$ subspace (shown in Fig. 1) appear sufficient for their accurate description.

In short, we show that SA-NCSM yields results in reduced SU(3)-based model spaces that practically coincide with the ones obtained in the corresponding complete space. The computational complexity associated with such calculations is examined next along with exploration of memory savings achievable in larger spaces using the examples of ${ }^{6} \mathrm{Li}$ and ${ }^{12} \mathrm{C}$.

\section{COMPUTATIONAL COMPLEXITY}

Issues governing the required computational resources include the model space dimension, the number of nonvanishing many-body matrix elements and the computational effort required by those matrix elements [50-53]. For the highly scalable algorithms we have developed and implemented, the computational resources can be viewed primarily in terms of memory and time requirements. We now turn our attention to these computational complexity issues.

The nuclear many-body calculations we address here involve evaluating the Hamiltonian in a selected basis representation and solving the resulting matrix for a small set (typically less than 20) of its low-lying eigenvalues and eigenvectors. To solve the large sparse matrix for its eigenvalues and eigenvectors on a massively parallel architecture is recognized as computationally hard. The challenge in nuclear physics is compounded by the strong inter-nucleon interactions referred to in Eq. (1) 
[50] that induce significant short- and intermediate-range inter-nucleon correlations. The model space must therefore be sufficiently large to account for these correlations and, at the same time, account for the long-range "tails" of the nuclear wavefunctions. The need for accurate representations of the short, intermediate and long-range features of the wavefunctions drives the computational resources in different ways for the methods we employ. For example, the need for particular excited states (such as members of a rotational band) that lie in a region of high level density motivates the fixed- $J$ basis.

Each method we employ involves a transformation of stored $N N$ (and possibly $N N N$ ) matrix elements to the chosen many-nucleon basis representation. This process begins with initial interaction matrix elements stored in the HO basis that are reduced matrix elements, i.e. matrix elements in a basis coupled to total angular momentum $J$ (and sometimes total isospin $T$ ), and accommodate charge-dependent interactions. Currently, this initial interaction scheme is employed for both $N N$ and $N N N$ interactions but only $N N$ interactions are employed here.

The transformation of these initial interaction data sets to the representation of the basis of choice involves "recoupling transformations" that may be viewed as multiplications of 3 non-square matrices (see Ref. [67] for a graphical illustration). Our different choices of manynucleon basis (e.g., $M$-scheme basis, $J$-scheme basis, and $\mathrm{SU}(3)$-scheme basis) involve different recoupling transformations (see Ref. [32] for the SU(3) scheme). While for the NCSM, this transformation is part of the MFDn code, the SA-NCSM invokes a separate algorithm, which transforms each interaction set only once and stores it for use by the LSU3shell. Improving the efficiency of the recoupling transformations themselves and reducing their memory footprints are subjects of intensive ongoing research and involves, among other issues, efficient exploitation of computer architectures such as Graphics Processing Units. We will limit our considerations here to the computationally demanding evaluation of the manybody Hamiltonian and computation of the corresponding eigenvalues and eigenvectors.

Figure 5 presents model space dimensions (size of the many-body Hamiltonian matrix) in the $M$-scheme over a range of $N_{\max }$ values for even-even $N=Z$ nuclei. The figure illustrates the dramatic increase of matrix dimensions with increasing $N_{\max }$ and increasing atomic number $A$. For example, the corresponding ${ }^{12} \mathrm{C}$ data points show the size of the $M$-scheme basis used at each $N_{\max }$ to produce the results of Fig. 2. This increase in dimensionality and associated increase in the number of nonzero many-body matrix elements motivate the search for model spaces with the aim of reducing the computational complexity as also pursued by other efforts mentioned earlier [8, 37-39].

For modern realistic $N N$ and $N N N$ interactions, we have found for $p$-shell nuclei that $N_{\max }$ values of 8 and above are desirable for achieving results approaching con- vergence [40, 42-45, 68, 69]. The calculation of ${ }^{12} \mathrm{C}$ in the complete $N_{\max }=8$ model space by the MFDn takes between 10 and 15 minutes of wall-clock time using 124 nodes of Edison, a Cray XC30 system installed at the National Energy Research Scientific Computing Center. Each node of Edison has two 12-core Intel "Ivy Bridge" processors running at $2.4 \mathrm{GHz}$ and $64 \mathrm{~GB}$ of main memory. Namely, it takes less than 3 minutes to read and process all necessary input data, set up and distribute the $M$-scheme basis, determine the sparsity structure, and generate the nonzero matrix elements. Subsequently, each Lanczos iteration takes about 1.2 seconds. The final time depends on the number of eigenstates, and which (if any) observables are calculated for these eigenstates. Typically, several hundred iterations are needed to converge the lowest 10 states.

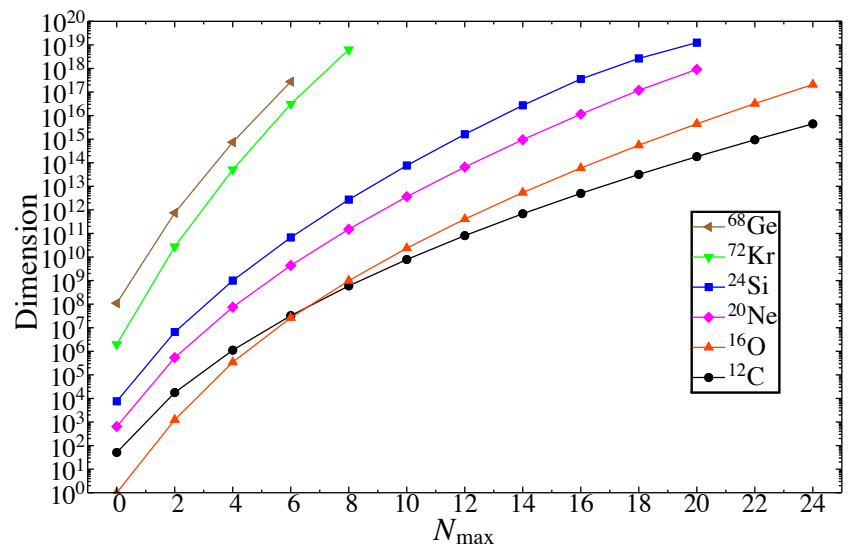

FIG. 5: Dimensions of the $M$-scheme (with $M=0$ ) natural parity model spaces for the even-even $N=Z$ nuclei with $N_{\max }$ truncation. The sequence of dimensions for unnatural parity states (odd values of $N_{\max }$ ) lie intermediate to the neighboring natural parity dimensions.

SA-NCSM runtime and number of single-shell configurations. - Computing the nuclear many-body Hamiltonian matrix in an SU(3) symmetry-adapted basis within the SA-NCSM framework represents the most computationally intensive task - one that typically takes over $95 \%$ of the total CPU time. Fortunately, this task can be considered a perfectly parallel problem and endows LSU3shell code with good scalability to hundreds of thousands of cores and possibly even beyond (Fig. 6). Each MPI process is assigned a submatrix of the Hamiltonian and invokes OpenMP threads to share the workload. The communication network is used merely to distribute data between collaborating processes during the initial set-up phase. No other communication between processes is needed, as each matrix element of the many-body Hamiltonian matrix can be evaluated independently. SU(3) basis states are mapped to the MPI processes in a block round-robin fashion, where the blocks are defined by similar SU(3) structures using the same set of $\mathrm{SU}(3)$ coupling coefficients and reduced ma- 
trix elements needed to evaluate Hamiltonian matrix elements, as described below. This approach leads to a uniform distribution of matrix elements and thus allows one to achieve a reasonably good load balancing.

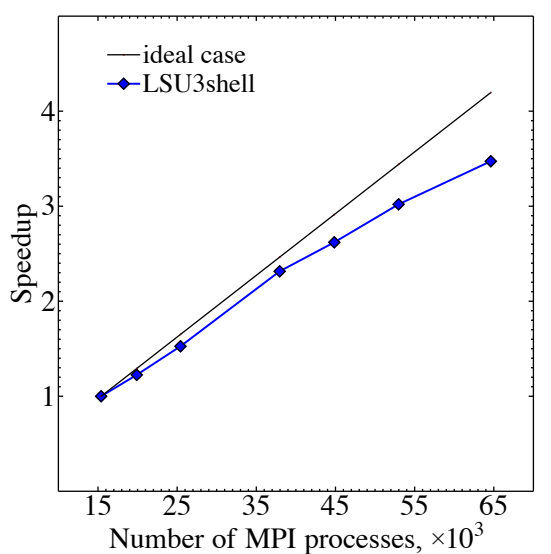

(a)

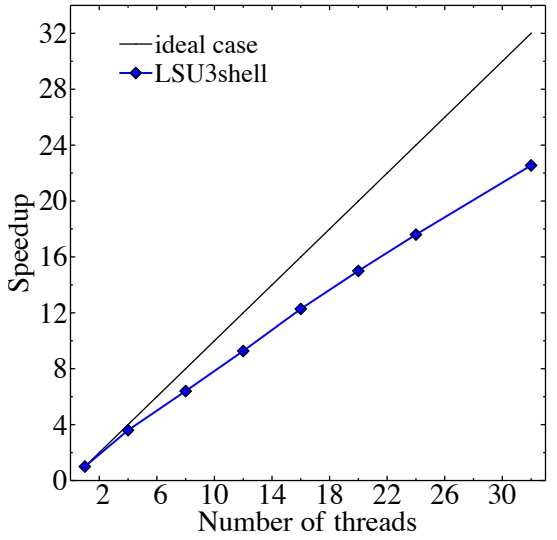

(b)

FIG. 6: (a) Strong scaling of the Hamiltonian matrix construction for $J=0$ states of ${ }^{24} \mathrm{Mg}$ (blue curve, dim = $\left.0.98 \times 10^{6}\right)$ as a function of the number of MPI processes (in units of thousands). (b) Strong scaling of LSU3shell on a single Cray XE6 compute node as a function of the number of OpenMP threads. (Scaling results are obtained on the Blue Waters system.)

The SA-NCSM implements fast methods for calculating matrix elements of arbitrary operators in the symmetry-adapted basis. This facilitates both the evaluation of the Hamiltonian matrix elements and the use of the resulting eigenvectors to evaluate other experimental observables. The underlying principle behind the SA-NCSM computational kernel is an SU(3)-type Wigner-Eckhart theorem, which factorizes interaction matrix elements into the product of $\mathrm{SU}(3)$ reduced matrix elements (RME) and the associated $\mathrm{SU}(3)$ coupling coefficients. To compute the Hamiltonian matrix elements, LSU3shell adopts state-of-the-art grouptheoretical methods [62], implemented in $\mathrm{C}++$ programming language, and optimized Fortran numeri- cal subroutines [70] for computing required $\mathrm{SU}(3)$ coupling/recoupling coefficients. As described above, the SA-NCSM configurations are constructed as the intershell coupling of a set of single-shell irreps of $\mathrm{U}\left(\Omega_{\eta}\right) \times$ $\mathrm{SU}(2)_{S}$ with $\mathrm{U}\left(\Omega_{\eta}\right) \supset \mathrm{SU}(3)$. Therefore, all the multishell RME are constructed from a set of single-shell RME computed in a configuration space of the single-shell irreps. This reduces the number of key pieces of information required to the single-shell RME, and these track with the number of the single-shell $\mathrm{U}\left(\Omega_{\eta}\right) \times \mathrm{SU}(2)_{S}$ irreps, with $\mathrm{U}\left(\Omega_{\eta}\right) \supset \mathrm{SU}(3)$, that represent building blocks of the SA-NCSM approach. It is therefore significant that their number grows slowly with the increasing nucleon number and $N_{\max }$ cutoff as illustrated in Fig. 7, as this allows these key pieces of information to be stored in CPU memory in the LSU3shell code. The baseline implementation of the algorithm for computing manynucleon matrix elements in an $\mathrm{SU}(3)$ basis takes, e.g., in case of $\langle 6\rangle 8$-A space of $J=0$ states in ${ }^{12} \mathrm{C}, 17$ minutes of wall-clock time to compute $10^{11}$ non-vanishing matrix elements using 124 nodes of Edison. The main performance bottleneck of the current implementation is its dependence on a frequent searching for computationally expensive data, e.g., SU(3) coupling/recoupling coefficients, stored in the computer's memory in look-up data structures, which lack spatial and temporal locality. This leads to a random memory access pattern with an inherently suboptimal utilization of hierarchical memory subsystems. Overcoming this challenge is currently a subject of ongoing research, in which we aim to find an optimal ordering of proton/neutron basis configurations and tensor operators that maximizes the reuse of computationally expensive data and evades a frequent random memory access in the computer's global memory.

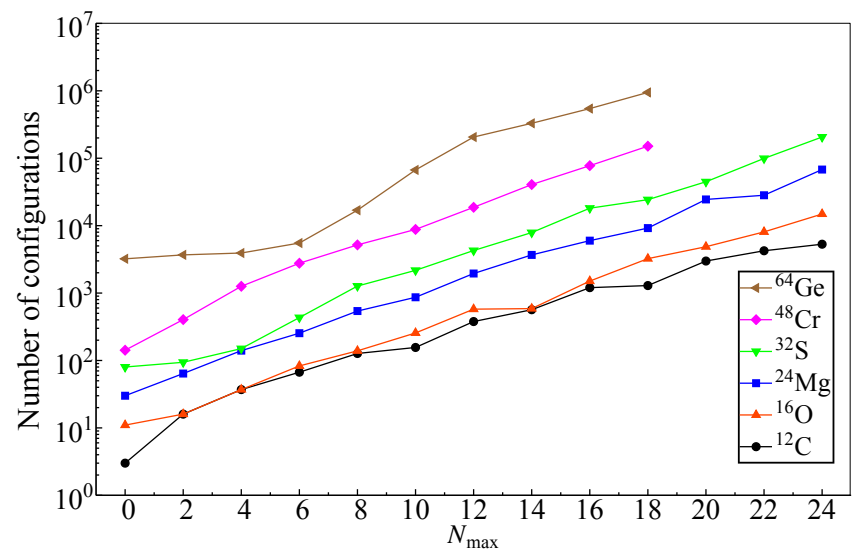

FIG. 7: Number of single-shell $\mathrm{U}\left(\Omega_{\eta}\right) \times \mathrm{SU}(2)_{S}$ irreps, with $\mathrm{U}\left(\Omega_{\eta}\right) \supset \mathrm{SU}(3)$, that generate the SA-NCSM model space for even-even $N=Z$ nuclei as a function of $N_{\max }$ cutoff.

An important feature of the SA-NCSM is that the symmetry-guided organization of the model space allows the full space to be systematically down-selected to the physically relevant subspace. As a second illustrative ex- 
ample, we consider SA-NCSM results for ${ }^{6} \mathrm{Li}$ low-lying states that, as shown in Ref. [18], have achieved significantly reduced dimensions for equivalent large shellmodel spaces without compromising the accuracy of the $a b$ initio NCSM approach (see Fig. 2 and Fig 3 of Ref. [18] for $g s$ and excitation energies, $B(E 2)$ values and $Q$ electric quadrupole moments). The runtime of the SANCSM code exhibits a quadratic dependence on the number of $(\lambda \mu)$ and $\left(S_{p} S_{n} S\right)$ irreps - there are $1.74 \times 10^{6}$ such irreps for the complete $N_{\max }=12$ model space of ${ }^{6} \mathrm{Li}$, while only $8.2 \%, 8.3 \%, 8.9 \%, 12.7 \%$, and $30.6 \%$ of these are retained when keeping all allowed irreps at $N_{\max }^{\perp}=2,4,6,8$, and 10 , respectively. The net result is that calculations in the $\langle 10\rangle 12,\langle 8\rangle 12, \ldots,\langle 2\rangle 12$ spaces require one to two orders of magnitude less computational time than SA-NCSM calculations for the complete $N_{\max }=12$ space.

Number of non-vanishing many-body Hamiltonian matrix elements - A very small fraction of the SA-NCSM runtime is devoted to the eigenvalue procedure. Since MFDn as well as the current implementation of LSU3shell, which utilizes the Lanczos parallel eigensolver of MFDn, store the non-vanishing many-body Hamiltonian matrix elements, the number of these elements in a specified basis dominates the storage requirements in our applications. For example, the number of the non-vanishing matrix elements in the $\langle 6\rangle 8$-B space are less or up to an order of magnitude greater than the $M$ scheme counterparts, while less than the $J$-scheme ones, as shown in Fig. 8 (see the $N_{\max }=8$ entries) for ${ }^{6} \mathrm{Li}$ (observables obtained in the model spaces discussed here for ${ }^{6} \mathrm{Li}$ are presented in Ref. [18]) and in Table II for ${ }^{12} \mathrm{C}$. The ${ }^{12} \mathrm{C}$ scenario probably realizes the complexity upper limit of the $\mathrm{SU}(3)$ scheme, since it is actually an exception rather than a typical performance. The reason is that the dominant deformation in ${ }^{12} \mathrm{C}$ is oblate and this results in relevant subspaces that usually consist of less deformed configurations of comparatively larger dimensionality. A more typical performance is expected for a nucleus like ${ }^{6} \mathrm{Li}$ (Fig. 8) with low-lying states of a prolate dominant deformation, which is the deformation favored by most nuclei.

Above all, as shown in Fig. 8, an important feature of the SA-NCSM is that the number of the non-vanishing matrix elements shows a slower increase with $N_{\max }$, as compared to the $J$ and $M$ schemes. Clearly, this number for the ' $\mathrm{B}$ ' selection is $50 \%, 17 \%, 8 \%$, and $4 \%$ of the corresponding $J$-scheme calculations in the complete $N_{\max }$ $=8,10,12$, and 14 basis (it is $38 \%, 14 \%, 7 \%$, and $3.5 \%$ compared to the $M$-scheme basis). This indicates that model spaces that are currently accessible on modern-day computer architectures can reach higher $N_{\max }$ within the SA-NCSM framework (e.g., $\langle 2\rangle 6$ for ${ }^{24} \mathrm{Si}[71]$ ). Such an efficient computational scaling with $N_{\max }$ is essential for the applicability of the SA-NCSM to larger model spaces needed to address largely deformed and spatial cluster configurations.

Dimension of model space (Hamiltonian matrix)

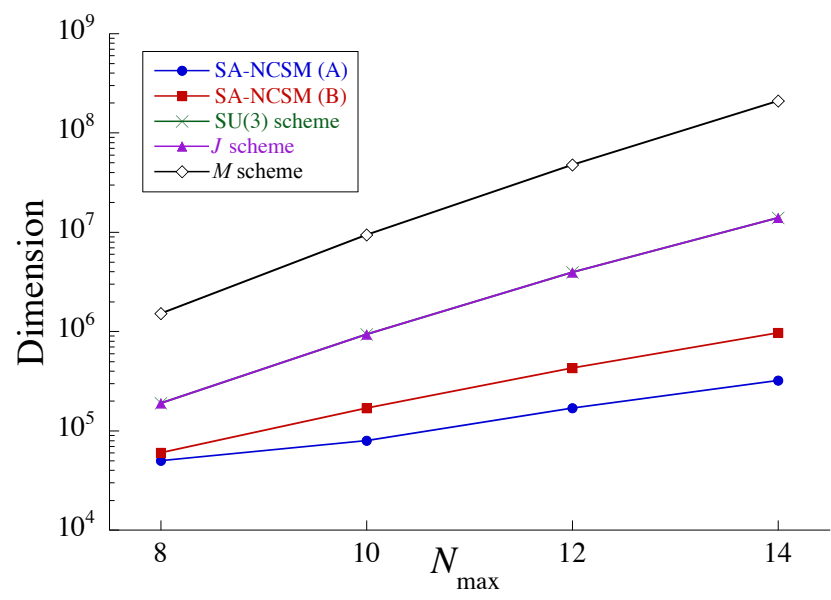

(a)

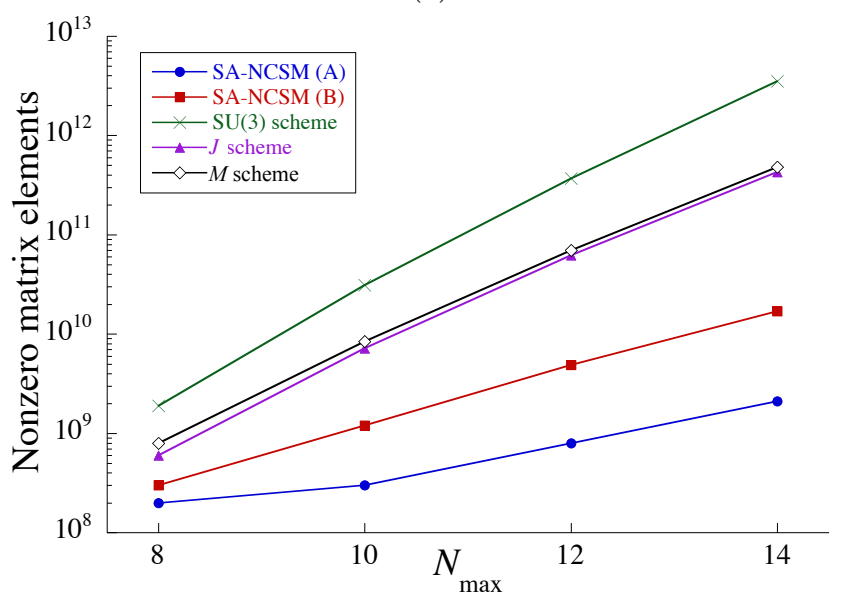

(b)

FIG. 8: (a) Matrix dimensions and (b) number of non-zero (nnz) many-body matrix elements for ${ }^{6} \mathrm{Li}$ and $J=1$ as functions of the $N_{\max }$ cutoff for selected SU(3) model spaces $\langle 6\rangle N_{\max }-\mathrm{A},\langle 6\rangle N_{\max }-\mathrm{B}$, and for the complete $N_{\max }$ space for the $\mathrm{SU}(3), J$, and $M$ schemes. The dimension for the $J$ scheme coincides with that for the $\mathrm{SU}(3)$ scheme; the total $M$-scheme dimension is shown for all $J \geq 1$ (for $M=1$ ). The nnz entries are quoted for an input $N N$ interaction.

- Analysis of results reveals that the SA-NCSM accurately reproduces corresponding complete-space results by using only a fraction of the model space. For example, the $\langle 6\rangle 8$-A $(\langle 6\rangle 8-\mathrm{B})$ selection for ${ }^{12} \mathrm{C}$ (Table II) produces dimensions that range from $10 \%$ to $15 \%$ (from $21 \%$ to $27 \%$ ) of the corresponding $J$-scheme NCSM basis dimensions as well as from $0.5 \%$ to $1.6 \%$ (from $0.7 \%$ to $3.4 \%$ ) of the corresponding $N_{\max }=8 M$-scheme NCSM basis dimensions (similar fractions are found for ${ }^{6} \mathrm{Li}$ ). The smaller number of basis states needed in the SA-NCSM for a successful description of these nuclear states points to a faster rate of convergence achieved in the symmetryadapted framework, as shown in Refs. [18] and [63]. The drastically reduced model spaces that are found sufficient to describe nuclear properties of $p$-shell nuclei confirm the 
TABLE II: Matrix dimensions ("dim", first row for each $J$ ) and number of non-vanishing many-body matrix elements ("nnz", second row for each $J$ ) for ${ }^{12} \mathrm{C}$ at selected values of total angular momentum and their dependence on the model space for $N_{\max }=8$. For reference, the total $M$-scheme dimension at $N_{\max }=8$ is $5.9 \times 10^{8}$ and the total number of non-vanishing matrix elements in this basis is $4.95 \times 10^{11}$. The dimension in the complete space is independent of the coupling scheme. The nnz entries are quoted for an input $N N$ interaction and, for the $J=1$ and $J=4 J$-scheme entries, are estimates that should be accurate to better than $10 \%$.

\begin{tabular}{|c|c|c|c|c|}
\hline \multirow[b]{2}{*}{$J$} & \multicolumn{2}{|c|}{$\langle 6\rangle 8$} & \multicolumn{2}{|c|}{ complete space } \\
\hline & $\mathrm{A}$ & B & $\mathrm{SU}(3)$ & $J$-scheme \\
\hline \multirow{2}{*}{$J=0$} & $2.8 \times 10^{6}$ & $4.0 \times 10^{6}$ & $1.9 \times 10^{7}$ & $1.9 \times 10^{7}$ \\
\hline & $1.0 \times 10^{11}$ & $1.7 \times 10^{11}$ & $2.5 \times 10^{12}$ & $9.5 \times 10^{11}$ \\
\hline \multirow{2}{*}{$J=1$} & - & $1.4 \times 10^{7}$ & $5.4 \times 10^{7}$ & $5.4 \times 10^{7}$ \\
\hline & - & $1.9 \times 10^{12}$ & $2.0 \times 10^{13}$ & $1.3 \times 10^{13}$ \\
\hline \multirow{2}{*}{$J=2$} & $1.0 \times 10^{7}$ & $1.6 \times 10^{7}$ & $7.9 \times 10^{7}$ & $7.9 \times 10^{7}$ \\
\hline & $1.2 \times 10^{12}$ & $1.6 \times 10^{12}$ & $2.2 \times 10^{13}$ & $1.7 \times 10^{13}$ \\
\hline \multirow{2}{*}{$J=4 \underset{\mathrm{nnz}}{\operatorname{dim}}$} & $9.4 \times 10^{6}$ & $2.0 \times 10^{7}$ & $9.1 \times 10^{7}$ & $9.1 \times 10^{7}$ \\
\hline & $1.7 \times 10^{12}$ & $3.9 \times 10^{12}$ & $3.9 \times 10^{13}$ & $3.1 \times 10^{13}$ \\
\hline
\end{tabular}

physical relevance of the $\mathrm{SU}(3)$ basis for nuclear modeling.

In short, we have shown that solutions of the SANCSM with symmetry-dictated model-space selections retain accuracy and are highly scalable on massively parallel architectures. They also require computations for significantly reduced model-space dimensions and $\mathrm{nnz}$ compared with the fixed- $J$ basis of NCSM while enhancing access to converged nuclear properties.

\section{SUMMARY}

We have demonstrated the efficacy of the SU(3)scheme that utilizes symmetries to reduce the dimensionality of the model space and, more importantly, the number of non-zero many-body matrix elements through a very structured selection of the basis states to physically relevant subspaces without compromising the accuracy of the $a b$ initio NCSM approach.

While applications of the NCSM and SA-NCSM in complete model spaces necessarily yield identical results (indeed, this is how the SA-NCSM implementation was validated), the intended use of the two approaches is complementary. The NCSM offers the possibility to produce complete-space results for light nuclei; the SA-NCSM is intended for applications beyond the lightest nuclei and to cluster formation where deformed structures are known to dominate the nuclear landscape and symmetryguided selections are essential. In addition, the SANCSM is suitable for applications to collective states and rotational bands, including those that lie in high leveldensity regions of nuclear spectra. In particular, the SANCSM enables one to reach beyond currently accessible model spaces, which are complete through an $N_{\max }$ truncation, by reducing such model spaces and augmenting them with configurations beyond $N_{\max }$ that are necessary to describe collective modes. We have shown that the current implementation of the SA-NCSM, LSU3shell, is highly scalable and efficiently manages the added computational time associated with tracking symmetries. Moreover, it exhibits reasonable scaling of the associated memory requirement with $N_{\max }$. These features make SANCSM calculations feasible on present massively parallel computer architectures. This, in turn, opens the path toward no-core shell-model descriptions of largely deformed nuclear states and cluster substructures as well as heavier nuclei.

\section{ACKNOWLEDGMENTS}

We acknowledge useful discussions with B. R. Barrett and A. M. Shirokov, as well as with D. J. Rowe and C. Bahri. We are grateful to E. Ng and C. Yang for developments leading to the $J$-scheme statistics for Table II and Fig. 8. This work was supported in part by NSF grants OCI-0904874, OCI-0904782, and OCI-0904809. In addition, this research was supported in part by US DOE Grants DE-SC0005248, DEFG02-95ER-40934, DESC0008485 (SciDAC/NUCLEI) and DE-FG02-87ER40371, by the Czech Science Foundation under Grant No. 16-16772S, by the Southeastern Universities Research Association, and by the US DOE, Office of Advanced Scientific Computing Research, through the Ames Laboratory, operated by Iowa State University under contract No. DE-AC02-07CH11358. A portion of the computational resources were provided by the National Energy Research Scientific Computing Center, which is supported by the Office of Science of US DOE under Contract No. DE-AC02-05CH11231, and by an INCITE award, "Nuclear Structure and Nuclear Reactions", from the DOE Office of Advanced Scientific Computing. This research also used resources of the Oak Ridge Leadership Computing Facility at ORNL, which is supported by the DOE Office of Science under Contract DE-AC05-00OR22725. This work is also part of the "Collaborative Research: Innovative ab initio symmetryadapted no-core shell model for advancing fundamental physics and astrophysics" PRAC allocation support by NSF (award number ACI-1516338), and is part of the Blue Waters sustained-petascale computing project, which is supported by NSF (awards OCI-0725070 and ACI-1238993) and the state of Illinois (Blue Waters is a joint effort of the University of Illinois at UrbanaChampaign and its National Center for Supercomputing Applications). Portions of this research were conducted with high performance computing resources provided by Louisiana State University (http://www.hpc.lsu.edu). 
[1] S. C. Pieper, R. B. Wiringa, and J. Carlson, Phys. Rev. C 70, 054325 (2004).

[2] K. M. Nollett, S. C. Pieper, R. B. Wiringa, J. Carlson, and G. M. Hale Phys. Rev. Lett. 99, 022502 (2007).

[3] S. C. Pieper, La Rivista del Nuovo Cimento, 31, 709 (2008) [arXiv:0711.1500v1 [nucl-th]].

[4] P. Navrátil, J. P. Vary, and B. R. Barrett, Phys. Rev. Lett. 84, 5728 (2000); Phys. Rev. C 62, 054311 (2000).

[5] S. Quaglioni and P. Navrátil, Phys. Rev. Lett. 101, 092501 (2008); Phys. Rev. C 79, 044606 (2009).

[6] B. R. Barrett, P. Navrátil and J. P. Vary, Prog. Part. Nucl. Phys. 69, 131 (2013).

[7] G. Hagen, T. Papenbrock and M. Hjorth-Jensen, Phys. Rev. Lett. 104, 182501(2010).

[8] R. Roth and P. Navrátil, Phys. Rev. Lett. 99, 092501 (2007).

[9] E. Epelbaum, H. Krebs, D. Lee, and Ulf-G. Meissner, Phys. Rev. Lett. 106, 192501 (2011).

[10] E. Epelbaum, W. Glöckle, and Ulf-G. Meissner, Nucl. Phys. A 637, 107 (1998); 671, 295 (2000).

[11] D. R. Entem and R. Machleidt, Phys. Rev. C 68, 041001(R) (2003).

[12] R. Machleidt and D. R. Entem, Phys. Rept. 503, 1 (2011).

[13] R. B. Wiringa, V. G. J. Stoks and R. Schiavilla, Phys. Rev. C 51, 38 (1995) [arXiv:nucl-th/9408016].

[14] S. C. Pieper, V. R. Pandharipande, R. B. Wiringa, and J. Carlson, Phys. Rev. C 64, 014001 (2001)

[15] S. C. Pieper, AIP Conf. Proc. 1011, 143(2008).

[16] A. M. Shirokov, J. P. Vary, A. I. Mazur and T. A. Weber, Phys. Letts. B 644, 33 (2007).

[17] A. M. Shirokov, J. P. Vary, A. I. Mazur, S. A. Zaytsev and T. A. Weber, Phys. Lett. B 621, 96 (2005); subroutines to generate this interaction in the relative-center-of-mass HO basis are available at nuclear.physics.iastate.edu.

[18] T. Dytrych, K. D. Launey, J. P. Draayer, P. Maris, J. P. Vary, E. Saule, U. Çatalyürek, M. Sosonkina, D. Langr, and M. A. Caprio, Phys. Rev. Lett. 111, 252501 (2013).

[19] J. P. Elliott, Proc. Roy. Soc. A245, 128 (1958); ibid. A245, 562 (1958).

[20] J. P. Elliott and M. Harvey, Proc. Roy. Soc. A272, 557 (1962).

[21] G. Rosensteel and D. J. Rowe, Phys. Rev. Lett. 38, 10 (1977).

[22] D. J. Rowe, Rep. Prog. Phys. 48, 1419 (1985).

[23] M. Harvey, Advan. Nucl. Phys. 1, 67 (1968).

[24] G. Rosensteel and D. J. Rowe, Ann. Phys. N. Y. 126, 343 (1980).

[25] J. P. Draayer and K. J. Weeks, Phys. Rev. Lett. 51, 1422 (1983).

[26] J. P. Draayer, K. J. Weeks and G. Rosensteel, Nucl. Phys. A413, 215 (1984).

[27] Y. Suzuki and K. T. Hecht, Nucl. Phys. A455, 315 (1986).

[28] Y. Suzuki and S. Hara, Phys. Rev. C 39, 658 (1989).

[29] A. C. Dreyfuss, K. D. Launey, T. Dytrych, J. P. Draayer, and C. Bahri, Phys. Lett. B 727, 511 (2013).

[30] G. K. Tobin, M. C. Ferriss, K. D. Launey, T. Dytrych, J. P. Draayer, A. C. Dreyfuss, and C. Bahri, Phys. Rev. C 83, 034312 (2014).
[31] K. D. Launey, A. C. Dreyfuss, J. P. Draayer, T. Dytrych, and R. Baker, J. Phys. Conf. Ser. 569, 012061 (2014).

[32] K. D. Launey and T. Dytrych and J. P. Draayer and G.H. Sun and S.-H. Dong, Int. J. Mod. Phys. E 24, 1530005 (2015).

[33] T. Dytrych, A. C. Hayes, K. D. Launey, J. P. Draayer, P. Maris, J. P. Vary, D. Langr, and T. Oberhuber, Phys. Rev. C 91, 024326 (2015).

[34] T. Dytrych, K. D. Sviratcheva, C. Bahri, J. P. Draayer and J. P. Vary, Phys. Rev. Lett. 98, 162503 (2007).

[35] T. Dytrych, K. D. Sviratcheva, C. Bahri, J. P. Draayer, and J. P. Vary, Phys. Rev. C 76, 014315 (2007).

[36] T. Dytrych, K. D. Sviratcheva, J. P. Draayer, C. Bahri and J. P. Vary, J. Phys. G. 35, 123101 (2008).

[37] T. Otsuka, T. Mizusaki and M. Honma, J. Phys. G: Nucl. Part. Phys. 25, 699 (1999).

[38] T. Otsuka, M. Honma, T. Mizusaki, N. Shimizu, and Y. Utsuno, Prog. Part. Nucl. Phys. 47, 319 (2001).

[39] T. Abe, P. Maris, T. Otsuka, N. Shimizu, Y. Utsuno and J. P. Vary, Phys. Rev. C 86, 054301 (2012); [arXiv:1204.1755 [nucl-th]].

[40] P. Maris, J. P. Vary, A. M. Shirokov, Phys. Rev. C 79, 014308 (2009).

[41] P. Maris, A. M. Shirokov and J. P. Vary, Phys. Rev. C 81, 021301(R) (2010).

[42] P. Maris and J. P. Vary, Int. J. Mod. Phys. E 22, 1330016 (2013).

[43] A. M. Shirokov, V. A. Kulikov, P. Maris and J. P. Vary, Nucleon-Nucleon and Three Nucleon Interactions, edited by L.D. Blokhintsev and I.I. Strakovsky, (Nova Science, 2014), Chapter 8, p. 231.

[44] S. A. Coon, M. I. Avetian, M. K. G. Kruse, U. van Kolck, P. Maris and J. P. Vary, Phys. Rev. C 86, 054002 (2012) [arXiv:1205.3230 [nucl-th]].

[45] R. J. Furnstahl, G. Hagen and T. Papenbrock, Phys. Rev. C 86, 031301 (2012) [arXiv:1207.6100 [nucl-th]].

[46] S. Konig, S. K. Bogner, R. J. Furnstahl, S. N. More, and T. Papenbrock, Phys. Rev. C 90, 064007 (2014).

[47] K. A. Wendt, C. Forssen, T. Papenbrock, and D. Saaf, Phys. Rev. C 91, 061301(R) (2015).

[48] D. Odell, T. Papenbrock, and L. Platter, [arXiv:1512.04851 [nucl-th]].

[49] J. P. Vary, "The Many Fermion Dynamics Shell Model Code," Iowa State University, 1992 (unpublished); J. P. Vary and D. C. Zheng, ibid 1994 (unpublished).

[50] P. Sternberg, E. G. Ng, C. Yang, P. Maris, J. P. Vary, M. Sosonkina and H. V. Le, In Proc. 2008 ACM/IEEE Conf. on Supercomputing (Austin, Texas, November 1521, 2008).

[51] J. P. Vary, P. Maris, E. Ng, C. Yang and M. Sosonkina, J. Phys. Conf. Ser. 180, 12083 (2009).

[52] P. Maris, M. Sosonkina, J. P. Vary, E. G. Ng and C. Yang, ICCS 2010, Procedia Computer Science 1, 97 (2010).

[53] H.M. Aktulga, C. Yang, E.G. Ng, P. Maris, and J.P. Vary, Concurrency Computat.: Pract. Exper. 26, 2631 (2013); doi: $10.1002 /$ cpe.3129.

[54] H. M. Aktulga, C. Yang, E. G. Ng, P. Maris and J. P. Vary, in 2011 International Conference on High Performance Computing and Simulation (HPCS), 176, (2011); doi: 10.1109/HPCSim.2011.5999822

[55] T. Dytrych, "LSU3shell code", Louisiana State Uni- 
versity, available under the GNU General Public License at the git repository, http://sourceforge.net/ projects/lsu3shell.

[56] H. J. Lipkin, Phys. Rev. 109, 2071 (1958).

[57] B. J. Verhaar, Nucl. Phys. A 21, 508 (1960).

[58] D. H. Gloeckner and R. D. Lawson, Phys. Lett. B 53, 313 (1974).

[59] O. Castaños, J. P. Draayer and Y. Leschber, Z. Phys 329, 33 (1988).

[60] G. Rosensteel and D. J. Rowe, Ann. Phys. N.Y. 104, 134 (1977).

[61] Y. Leschber and J. P. Draayer, Phys. Letts. B 190, 1 (1987).

[62] J. P. Draayer, Y. Leschber, S. C. Park, and R. Lopez, Comput. Phys. Comm. 56, 279 (1989).

[63] T. Dytrych et al., to be submitted (2016).

[64] F. Ajzenberg-Selove and J. Kelley, Nucl. Phys. A 5061 (1990)
[65] I. Tanihata et al., Phys. Rev. Lett. 55, 2676 (1985).

[66] P. Maris et al., J. Phys. Conf. Ser. 403, 012019 (2012).

[67] D. Oryspayev, H. Potter, P. Maris, M. Sosonkina, J. P. Vary, S. Binder, A. Calci, J. Langhammer and R. Roth, IEEE 27th Parallel and Distributed Processing Symposium Workshops \& PhD Forum (IPDPSW), 1365 (2013); DOI: 10.1109/IPDPSW.2013.253.

[68] P. Maris, J. P. Vary and P. Navrátil, Phys. Rev. C 87, 014327 (2013); [arXiv: 1205.5686].

[69] E. D. Jurgenson, P. Maris, R. J. Furnstahl, P. Navrátil, W. E. Ormand, J. P. Vary, Phys. Rev. C 87, 054312 (2013).

[70] Y. Akiyama and J. P. Draayer, Comput. Phys. Commun., 5, 405 (1973).

[71] J. P. Draayer, T. Dytrych, K. D. Launey, A. C. Dreyfuss, and D. Langr, J. of Phys. Conf. Ser. 578, 012010 (2015). 